\title{
Advances in Laparoscopy
}

\section{Single Stage Conversion from Laparoscopic Adjustable Gastric Band to Laparoscopic Sleeve Gastrectomy is Safer than Laparoscopic Adjustable Gastric Band to Roux-en-Y Gastric Bypass: Results of a 10-Year Analysis of Complications}

Raelina S. Howell, MD', Meredith Akerman, MS', Patrizio Petrone, MD, MPH, MHSA, FACS , Javier Perez-Calvo, $M D^{1}$, Harika Boinpally, $M D^{1}$, Jon $S$ Woods, $M D^{1}$, Keneth Hall, MD, FACS, FASMBS ${ }^{1}$, Alexander Barkan, MD, FACS, FASMBS ${ }^{1}$ and Collin EM Brathwaite, MD, MS, FACS, FASMBS $^{1^{*}}$

${ }^{1}$ Department of Surgery, NYU Winthrop Hospital, USA

${ }^{2}$ Department of Biostatistics, NYU Winthrop Hospital, USA

\begin{abstract}
Background: Conversion from laparoscopic Adjustable Gastric Band (AGB) to Roux-en-y gastric bypass (RNY) or Sleeve Gastrectomy (SG) is now common. Complication rates up to $25 \%$ have been reported. We reviewed the experience at a Metabolic and Bariatric Surgery Accreditation and Quality Improvement Program (MBSAQIP) Center of Excellence.

Study Design: Our prospective database was retrospectively reviewed for single-stage conversions over a 10-year period ending in 2017 to assess outcomes compared to primary procedures. In revisions, we reinforce staple lines with buttress material, oversew staple lines in high-risk patients, and avoid stapling or anastomosing into scar capsule tissue. Statistical analysis was performed using t-test, chi-square, Fisher's Exact, McNemar's, and Wilcoxon signed rank tests.
\end{abstract}

Results:

\begin{tabular}{|l|l|l|l|l|}
\hline \multicolumn{5}{|c|}{ Results } \\
\hline & $\begin{array}{l}\text { Primary RNY } \\
(\mathrm{n}=620)\end{array}$ & $\begin{array}{l}\text { Conversion to RNY } \\
(\mathrm{n}=50)\end{array}$ & $\begin{array}{l}\text { Primary SG } \\
(\mathrm{n}=1291)\end{array}$ & $\begin{array}{l}\text { Conversion to SG } \\
(\mathrm{n}=146)\end{array}$ \\
\hline Age (years), & 43.8 & 49.7 & 43.4 & 46.8 \\
\hline mean (range) & $(18-78)$ & $(24-67)$ & $(13-73)$ & $(23-73)$ \\
\hline Female, $\mathrm{n}(\%)$ & $464(74.8)$ & $44(88)$ & $986(76.4)$ & $128(87.7)$ \\
\hline BMI, mean (range) & $47.6(28.8-86.0)$ & $46.2(34-66.4)$ & $46.4(27.8-87.4)$ & $44.5(33.4-74)$ \\
\hline Bleed, $\mathrm{n}(\%)$ & $6(0.1)$ & 0 & $3(0.2)$ & $1(0.7)$ \\
\hline *Leak, $\mathrm{n}(\%)$ & $1(0.2)$ & $4(8)$ & $8(0.6)$ & 0 \\
\hline Stricture, $\mathrm{n}(\%)$ & $10(1.6)$ & 0 & $5(0.4)$ & $1(0.7)$ \\
\hline †30-day Reoperation, $\mathrm{n}(\%)$ & $16(2.6)$ & $4(8)$ & $8(0.6)$ & 0 \\
\hline 30-day Readmission, $\mathrm{n}(\%)$ & $25(4.0)$ & $4(8)$ & $30(2.3)$ & $3(2.1)$ \\
\hline
\end{tabular}

*Fisher's Exact test for conversion RNY vs. conversion SG leak $p=0.0039$

${ }^{\dagger}$ Fisher's Exact test for conversion RNY vs. conversion SG re-op $p=0.0039$

Conclusions: The risk of leak appears to be higher with conversion to RNY vs. SG. This finding should be weighed heavily when selecting revision procedures for high-risk patients.

Keywords

Bariatric Conversion, Roux-En-Y Gastric Bypass, Sleeve Gastrectomy, Adjustable Gastric Band, Bariatric Conversion Complications, MBSAQIP

Copyright: (C) 2020 Howell RS, et al. This is an open-access article distributed under the terms of the Creative Commons Attribution License, which permits unrestricted use, distribution, and reproduction in any medium, provided the original author and source are credited. 
Citation: Howell RS, Akerman M, Petrone P, et al. (2020) Single Stage Conversion from Laparoscopic Adjustable Gastric Band to Laparoscopic Sleeve Gastrectomy is Safer than Laparoscopic Adjustable Gastric Band to Roux-en-Y Gastric Bypass: Results of a 10-Year Analysis of Complications. Adv Laparoscopy 3(1):76-81

\section{Introduction}

Laparoscopic adjustable gastric banding (AGB), once the bariatric procedure par excellence, has diminished in popularity due to insufficient weight loss or band-related complications, which have been reported in up to $53 \%$ of cases $[1,2]$. Currently, many patients with AGB request conversion to another form of bariatric surgery. Conversion from AGB to sleeve gastrectomy (SG) or Roux-en-y gastric bypass (RNY) can be performed in one or two stages. In the single-stage procedure, the AGB components are removed and the gastric sleeve or bypass is created during the same operation. Comparatively, in the two-stage process the AGB hardware is removed in the first stage and the gastric sleeve or bypass is created in a separate, shorter, subsequent procedure [3]. However, prior studies did not find a significant difference in cumulative operating time between the one- and two-stage methods [4]. Single-stage conversion from AGB to SG or RNY has become a valid rescue, in spite of associated perioperative complications, such as stricture, bleeding, and increased length of stay [5].

Revisions and conversions have now become a significant part of bariatric surgery. According to the American Society for Metabolic and Bariatric Surgery (ASMBS), revisional bariatric surgery includes conversion, corrective, and reversal procedures [6]. Conversion is defined as a procedure that changes the index procedure to a different type. Corrective procedures are those that address complications or incomplete treatment effects of a previous bariatric procedure. Reversals are procedures that restore the original anatomy. However, information regarding surgical outcomes of revisional procedures can be inadvertently muddled. Due to the lack of consensus in the literature, we have defined revision as a recreation of the original procedure and conversion as a change from one procedure to another (eg AGB to SG). The objective of this study was to review the experience of single-stage conversions at a Center of Excellence in the Metabolic and Bariatric Surgery Accreditation and Quality Improvement Program (MBSAQIP). Our hypothesis is that single-stage conversions from $A G B$ to $S G$ or RNY are safe and feasible with a low complication rate.

\section{Methods}

After obtaining Institutional Review Board (IRB) approval, our prospective database was retrospectively reviewed for primary SG, primary RNY, and all single-stage bariatric conversions from AGB to SG or RNY performed over a 10-year period ending in 2017 to assess short-term outcomes. Factors reviewed included patient demographics (age, gender, weight, body mass index [BMI], co-morbidities), operative details (procedure performed, modality), and outcomes (length of hospital stay [LOS], bleed, leak, stricture, 30-day reoperation, 30-day readmission, 30-day mortality). Patients were categorized into two groups: those who underwent primary bariatric procedures and those who underwent conversion from AGB to RNY or SG. The decision to convert to either RNY or SG was largely based on patient preference. However, if patients were known to have severe gastroesophageal reflux disease (GERD), conversion to RNY was typically performed.
Also, if patients were known to have prior extensive mid-abdominal surgeries, conversion to SG was selected.

\section{Statistical Methods}

Since randomization of patients to primary or conversion could not be done due to the retrospective nature of the study, the AGB-to-SG and AGB-to-RNY conversion groups were compared to the primary SG and RNY cohorts, respectively, using the propensity score method (PSM). PSM was utilized to match patients in each of the two groups on the demographic variables gender, age, BMI, weight, and height in order to reduce the confounding effects of covariates on the targeted outcome variables. Descriptive statistics (mean \pm standard deviation and median for continuous data; frequencies and percentages for categorical data) were used to describe the demographic and clinical characteristics of the sample by group. Comparability of the two groups before and after matching was evaluated using the two sample t-test for continuous variables, and the chi-square test or Fisher's exact test, as deemed appropriate, for categorical variables.

McNemar's test for matched pair samples was used to compare the rates of the primary and secondary categorical outcomes (complications, comorbidities, 30-day readmission, and 30-day reoperation) for the two matched groups. Comorbidities were compared as outcomes in view of the fact that many patients presenting for conversion procedures may have had a significant change in the presence of comorbidities (e.g. resolution of diabetes, new-onset GERD). The Wilcoxon signed rank test was used to compare the two matched groups for continuous measures (i.e. LOS). A result was considered statistically significant at the $p<0.05$ level of significance. All analyses were performed using SAS version 9.4 (SAS Institute Inc., Cary, NC).

\section{Perioperative management}

This institution offers a "Back on Track" Program for patients seeking revision or conversion bariatric surgery for significant weight regain. Extensive preoperative assessments were performed, including nutritional and psychological evaluations. Smoking cessation was required for a minimum of six weeks prior to bariatric procedures and confirmatory testing was done using carboxyhemoglobin and nicotine levels. Non-compliant patients did not undergo elective operation. Oral contraceptive pills were held one month prior to and one month following surgery. Females of child-bearing age were

*Corresponding author: Collin EM Brathwaite, MD, FACS, FASMBS, Chairman, Department of Surgery, NYU Winthrop Hospital, 222 Station Plaza North, Suite 300, Mineola, NY, 11501, USA; Tel: +1-516-663-39, E-mail: collin.brathwaite@nyulangone.org

Accepted: September 26, 2020

Published online: September 28, 2020

Citation: Howell RS, Akerman M, Petrone P, et al. (2020) Single Stage Conversion from Laparoscopic Adjustable Gastric Band to Laparoscopic Sleeve Gastrectomy is Safer than Laparoscopic Adjustable Gastric Band to Roux-en-Y Gastric Bypass: Results of a 10-Year Analysis of Complications. Adv Laparoscopy 3(1):76-81 
Citation: Howell RS, Akerman M, Petrone P, et al. (2020) Single Stage Conversion from Laparoscopic Adjustable Gastric Band to Laparoscopic Sleeve Gastrectomy is Safer than Laparoscopic Adjustable Gastric Band to Roux-en-Y Gastric Bypass: Results of a 10-Year Analysis of Complications. Adv Laparoscopy 3(1):76-81

advised to avoid pregnancy for a minimum of 18 months postoperatively. Patients with a known hypercoaguable state were referred to hematology for evaluation prior to surgery. In patients with diabetes, insulin secretagogues were held and injectable insulin given accordingly. Prophylaxis against deep vein thrombosis included the preoperative use of one dose of 5000 units of low-molecular-weight heparin injected subcutaneously in combination with external, bilateral lower-extremity intermittent pneumatic compression. Postoperatively, daily enoxaparin sodium injections were used along with early and frequent ambulation. Patients with a BMI $>50$ $\mathrm{kg} / \mathrm{m}^{2}$ were also discharged with $40 \mathrm{mg}$ subcutaneous enoxaparin sodium twice a day for 14 days. Following a negative upper gastrointestinal swallow study (generally performed on postoperative day 1), patients were started on a low-sugar, clear liquid diet with supplemental crushed or liquid medications as necessary. Even though this institution has moved away from the use of routine postoperative upper gastrointestinal swallow studies following uncomplicated, primary bariatric procedures, they are still used for patients undergoing bariatric conversions due to the complexity and morbidity associated with the operation.

\section{Operative technique for conversions}

Conversion to sleeve gastrectomy: Patients were placed in a supine, then reverse-Trendelenburg position and five ports inserted into the abdomen $(12 \mathrm{~mm}$ left subcostal, 15 $\mathrm{mm}$ right upper quadrant, $5 \mathrm{~mm}$ bilateral subcostal, $5 \mathrm{~mm}$ subxiphoid). Adhesiolysis of the gastric band was performed using ultrasonic shears, followed by band unbuckling and extraction, along with removal of the tubing. The gastric fundoplication was then divided using sharp dissection with careful use of an energy device or with a stapler and articulating dissector if the stomach appeared thickened. Ultrasonic shears were then used to divide the gastrocolic omentum up to the angle of His. The orogastric tube was exchanged for a 38-French bougie, and the stomach divided using a stapler with buttress material (Peri-strips Dry ${ }^{\circledR}$ with Veritas ${ }^{\circledR}$, Baxter Healthcare Corporation) reinforcement. A thick-tissue black load was used for the first two staple firings in the antrum along the bougie, followed by a green load up to the angle of $\mathrm{His}$, crossing the staple lines to reduce the incidence of leak. Conversions performed at this institution include key steps in an effort to decrease morbidity: staple lines are over-sewn and/or reinforced with peri-strips in high-risk patients and precaution is taken to avoid stapling or anastomosing into AGB-induced scar capsule tissue. Special care was taken to staple away from the area of AGB scarring due to the relative ischemia of the fibrotic tissue. Finally, a leak test was performed by removing the bougie, replacing the orogastric tube, and instilling methylene blue at a high pressure to facilitate full distention of the stomach with close inspection of the staple line. For robotic cases, $8 \mathrm{~mm}$ robotic trocars were used in place of $5 \mathrm{~mm}$ laparoscopic ports. In cases when the robotic stapler was used, the staple line was over-sewn with 2-0 silk sutures in a seromuscular fashion.

Conversion to Roux-en-y gastric bypass: Patient positioning, port placement, band removal, and fundoplication takedown was performed in a similar fashion to that of the gastric sleeve procedure described above. The gastric pouch was then created using a buttress-reinforced stapler going up to the angle of His distal to the level of AGB-induced capsule to avoid incorporation of scar tissue into the staple line. The patient was repositioned from reverse-Trendelenburg to facilitate cranial passage of the ante-colic, ante-gastric Roux limb. The omentum was divided to the level of the transverse colon. The small bowel was then divided $50 \mathrm{~cm}$ distal to the ligament of Treitz, a $150 \mathrm{~cm}$ Roux limb was measured, and the respective anastomoses were created. Methylene blue was used to perform a leak test via an orogastric tube.

\section{Results}

The retrospective review revealed 2,816 patients who underwent bariatric surgery over the study period, 196 of whom were conversions from AGB. One hundred seventy-two $(87.8 \%)$ of the conversion patients were female. Fifty conversions were $A G B$ to RNY (25.5\%) and 146 were AGB to SG (74.5\%). Tables 1 and Table 2 show the patient demographics

Table 1: Patient demographics of the primary and conversion RNY groups.

\begin{tabular}{|c|c|c|c|}
\hline \multicolumn{4}{|c|}{ Patient Demographics } \\
\hline & \multicolumn{3}{|c|}{ RNY } \\
\hline & $1^{\circ}(n=50)$ & Conversion $(n=50)$ & $p$ \\
\hline Age (yrs), mean (SD) & 49 (12.78) & $50(11.15)$ & 0.6960 \\
\hline Female, $\mathrm{n}(\%)$ & $125(86.2)$ & 127 (87.6) & 0.7493 \\
\hline Weight (Ibs), mean (SD) & $267.9(48.2)$ & $275.5(48.44)$ & 0.4408 \\
\hline BMI, mean (SD) & $44.9(6.42)$ & $46.2(7.09)$ & 0.3419 \\
\hline HTN, n (\%) & $32(64)$ & $34(68)$ & 0.6729 \\
\hline DM, n (\%) & $20(40)$ & $13(26)$ & 0.1366 \\
\hline OSA, n (\%) & $28(56)$ & $22(44)$ & 0.1614 \\
\hline GERD, n (\%) & $32(64)$ & $33(66)$ & 0.8339 \\
\hline $\mathrm{OA}, \mathrm{n}(\%)$ & $26(52)$ & $25(50)$ & 0.8414 \\
\hline
\end{tabular}

BMI: Body mass index, HTN: Hypertension, DM: Diabetes mellitus, OSA: Obstructive sleep apnea, GERD: Gastroesophageal reflux disease, OA: Osteoarthritis. 
Citation: Howell RS, Akerman M, Petrone P, et al. (2020) Single Stage Conversion from Laparoscopic Adjustable Gastric Band to Laparoscopic Sleeve Gastrectomy is Safer than Laparoscopic Adjustable Gastric Band to Roux-en-Y Gastric Bypass: Results of a 10-Year Analysis of Complications. Adv Laparoscopy 3(1):76-81

Table 2: Patient demographics of the primary and conversion SG groups.

\begin{tabular}{|l|l|l|l|}
\hline \multicolumn{2}{|c|}{ Patient Demographics } \\
\cline { 2 - 4 } & \multicolumn{2}{|c|}{ SG } & P value \\
\hline Age (yrs), mean (SD) & $1^{\circ}(n=145)$ & Conversion ( $=145)$ & 0.6032 \\
\hline Female, $n$ (\%) & $46(11.91)$ & $47(11.56)$ & 0.7278 \\
\hline Weight (lbs), mean (SD) & $125(86.2)$ & $127(87.6)$ & 0.3391 \\
\hline BMI, mean (SD) & $260.9(44.65)$ & $266.1(47.13)$ & 0.2575 \\
\hline HTN, $n(\%)$ & $44(5.79)$ & $44.6(6.34)$ & 0.7242 \\
\hline DM, $n(\%)$ & $78(53.8)$ & $76(52.1)$ & 0.1402 \\
\hline OSA, $n(\%)$ & $43(29.7)$ & $32(21.9)$ & 0.1265 \\
\hline GERD, $n(\%)$ & $76(52.4)$ & $63(43.2)$ & 0.0003 \\
\hline OA, $n(\%)$ & $70(48.3)$ & $101(69.2)$ & 0.4661 \\
\hline
\end{tabular}

BMI: Body mass index, HTN: Hypertension, DM: Diabetes mellitus, OSA: Obstructive sleep apnea, GERD: Gastroesophageal reflux disease, OA: Osteoarthritis.

Table 3: Outcomes of primary versus conversion RNY procedures after propensity matching.

\begin{tabular}{|l|l|l|l|}
\hline \multicolumn{4}{|c|}{ Outcomes of Primary vs Conversion Procedures } \\
\hline & \multicolumn{1}{|c|}{ RNY } \\
\hline 30-day Readmission, $n(\%)$ & $1^{\circ}(n=50)$ & Conversion $(n=50)$ & P value \\
\hline 30-day Re-operation, $n$ (\%) & 0 & $4(8)$ & 0.1175 \\
\hline Mortality, $n$ (\%) & $2(4)$ & $4(8)$ & 0.6875 \\
\hline LOS (days), mean (SD) & 0 & 0 & N/A \\
\hline
\end{tabular}

Table 4: Outcomes of primary versus conversion SG procedures after propensity matching.

\begin{tabular}{|l|l|l|l|}
\hline \multicolumn{4}{|c|}{ Outcomes of Primary vs Conversion Procedures } \\
\hline & \multicolumn{1}{|c|}{ RNY } \\
\hline 30-day Readmission, $\mathrm{n}(\%)$ & $1^{\circ}(\mathrm{n}=50)$ & Conversion $(\mathrm{n}=50)$ & P value \\
\hline 30-day Re-operation, $\mathrm{n}(\%)$ & $4(2.8)$ & $3(2.1)$ & 1.0000 \\
\hline Mortality, $\mathrm{n}(\%)$ & 0 & 0 & N/A \\
\hline LOS (days), mean (SD) & 0 & 0 & N/A \\
\hline
\end{tabular}

for the conversion surgery patient groups compared to the historical control primary surgery groups after PSM.

Notably, prior to PSM the incidence of GERD was found to be significantly higher in patients undergoing either RNY or SG conversion compared to those undergoing primary RNY or SG. After PSM, the incidence of GERD was still found to be significantly higher in patients undergoing SG conversion compared to primary SG ( $p=0.0003$, chi-square). Additionally, the incidence of obstructive sleep apnea was found to be significantly lower in SG conversion patients compared to primary SG patients.

The RNY and SG conversion groups were also compared to each other. The mean age, weight, and BMI of the RNY conversion patients were not significantly different from the SG conversion patients ( $p=0.1007,0.2057$, and 0.1167 , respectively; unpaired t-test). The number of patients with hypertension was significantly higher in the RNY conversion group compared to the SG conversion group ( $p=0.0499$; chisquare). There was no difference in the number of patients with diabetes, obstructive sleep apnea, gastroesophageal reflux disease, or osteoarthritis between the RNY and SG conversion groups ( $p=0.5536,0.9167,0.6766,0.2045$, respectively; chi-square). Thirty-day outcomes were compared between the propensity-matched primary and conversion RNY (Table 3) and SG's (Table 4). The LOS was found to be significantly higher in patients who underwent conversion from AGB to SG compared to patients who underwent primary SG. Table 5 shows the comparison of the surgical outcomes of the RNY and SG conversion groups. The rate of leak, re-operation, and length of stay was found to be significantly lower in patients who underwent conversion to SG compared to those converted to RNY. The operative modality used was also found to be significantly different between the groups. 
Citation: Howell RS, Akerman M, Petrone P, et al. (2020) Single Stage Conversion from Laparoscopic Adjustable Gastric Band to Laparoscopic Sleeve Gastrectomy is Safer than Laparoscopic Adjustable Gastric Band to Roux-en-Y Gastric Bypass: Results of a 10-Year Analysis of Complications. Adv Laparoscopy 3(1):76-81

Table 5: Outcomes of conversion operations.

\begin{tabular}{|l|l|l|l|}
\hline \multicolumn{2}{|l|}{ Outcomes of Conversion Operation } & \multicolumn{3}{l|}{} \\
\hline & AGB to RNY Conversion $(\mathrm{n}=50)$ & AGB to SG Conversion ( $=146)$ & P value \\
\hline Modality, $\mathrm{n}(\%)$ Laparoscopic & $18(36)$ & $\mathbf{0 . 0 1 0 9}$ \\
\hline Robotic & $32(64)$ & $63(43)$ & $\mathbf{0 . 0 1 0 9}$ \\
\hline Time to conversion (months), mean (range) & $78(27-171)$ & $80(16-173)$ & 0.4783 \\
\hline Bleed, $\mathrm{n}(\%)$ & 0 & $1(0.7)$ & 1.0000 \\
\hline Leak, $\mathrm{n}(\%)$ & $4(8)$ & 0 & $\mathbf{0 . 0 0 3 9}$ \\
\hline Stricture, $\mathrm{n}(\%)$ & 0 & $1(0.7)$ & 1.0000 \\
\hline LOS (days), mean (range) & $3.3(1-22)$ & $2.4(1-13)$ & $\mathbf{0 . 0 0 1 0}$ \\
\hline 30-day Reoperation, $\mathrm{n}(\%)$ & $4(8)$ & 0 & $\mathbf{0 . 0 0 3 9}$ \\
\hline 30-day Readmission, $\mathrm{n}(\%)$ & $4(8)$ & $3(2.1)$ & 0.0717 \\
\hline Mortality, $\mathrm{n}$ (\%) & 0 & 0 & N/A \\
\hline
\end{tabular}

There were no mortalities in either conversion group.

\section{Discussion}

The ASMBS has published estimates of the number of bariatric procedures performed yearly in the United States from 2011-2017 [7]. Bariatric revisions (conversions, corrections, or reversals) accounted for $14 \%$ of the 228,000 total procedures performed in 2017 compared to only $6 \%$ in 2011 . Bariatric revision procedures are indicated for patients with inadequate weight loss, defined as less than $50 \%$ of excess weight loss at 18 months [8], or for patients with weight regain, comorbidity recurrence, or procedure-associated morbidities. Specific indications for reoperation after AGB placement include band slippage, erosion, port malfunction, or patient intolerance [9]. Patients can undergo band adjustment, replacement, or removal with or without conversion to another bariatric procedure. Conversion from a failed AGB to a SG or RNY can be performed in one- or two-stage procedure; however, there is currently no consensus in the literature regarding the optimal choice [6]. Major complications including leak and bleed are associated with both the single- and two-stage approach, with higher rates reported for the single-stage procedure $[4,10]$. However, LOS was shown to be longer for patients who underwent the two-stage operation [4]. Our single-stage complication rate may be low due to use of either staple line reinforcement with buttress material, over-sewing of the staple line or both in high risk patients, avoidance of stapling across the scar capsule left by the band, and placing the RNY anastomosis below the AGB-induced scar.

Complications of conversion to RNY or SG after AGB have been reported as high as $46 \%$ in previous studies [11-14]. In this study, single-stage conversion from AGB to RNY and SG did not appear to carry an increased risk of stricture, bleed, readmission, or death. However, the risk of leak appeared to be higher with conversion to RNY vs. SG. The decrease in morbidity could be due to the relatively decreased complexity of the SG versus the RNY operation (i.e. fewer anastomoses, less intra-abdominal manipulation). The LOS was also found to be lower in the SG conversion group and may also be associated with technical aspects of the procedure, though further studies are warranted. These findings should be weighed heavily when selecting revisional procedures for high-risk patients. Although RNY has been associated with greater longterm weight loss $[15,16]$, consideration must be given to the increased complication rate associated with conversion to RNY [5-17] when deciding to convert from AGB to either SG or RNY. Surgeons and patients, especially high-risk patients, should thoroughly discuss the potential pros and cons associated with each bariatric conversion procedure.

Bariatric surgery has been well-established to significantly reduce or even eliminate obesity-related comorbidities, such as diabetes, hypertension, obstructive sleep apnea, cardiovascular disease, and mortality [18-20]. In this study, the incidence of obstructive sleep apnea was found to be significantly lower in patients undergoing conversion to SG compared to primary SG. Recent reviews have specifically assessed the effect of bariatric surgery on GERD [21,22]. The authors reported that in the short-term postoperative period, gastric banding has been associated with alleviation of GERD symptoms. However, long-term follow up revealed a subset of patients who developed new-onset or worsening reflux, the etiology of which could be due directly to GERD or to band-related complications. In comparison, RNY has been associated with sustained resolution of GERD [21] and SG has been associated with worsening of GERD [23] or new-onset in up to $46 \%$ of patients [24]. Notably, the incidence of GERD was found to be significantly higher in patients prior to undergoing conversion to either RNY or SG compared to those about to undergo primary operation. The significant increase in GERD was also seen when comparing pre-primary SG to pre-conversion SG groups after propensity score matching. Consequently, the presence of GERD should be strongly taken into account when choosing revisional procedures.

The focus of our study was on short-term outcomes of patients undergoing conversion from primary AGB to RNY or SG at a Bariatric Center of Excellence. Future studies can assess the role of indocyanine green (ICG) and fluorescence imaging to visualize ischemic gastric tissue in relation to staple lines in order to potentially decrease the occurrence of leaks. Further studies are also underway to ascertain the long-term outcomes associated with single-stage conversions, as well as 
Citation: Howell RS, Akerman M, Petrone P, et al. (2020) Single Stage Conversion from Laparoscopic Adjustable Gastric Band to Laparoscopic Sleeve Gastrectomy is Safer than Laparoscopic Adjustable Gastric Band to Roux-en-Y Gastric Bypass: Results of a 10-Year Analysis of Complications. Adv Laparoscopy 3(1):76-81

a comparison to short- and long-term outcomes of two-stage procedures.

\section{Conclusions}

Single-stage conversion to either SG or RNY can be performed safely (either laparoscopically or robotically) in patients with a suboptimal response to AGB. Weight-loss goals and the presence of comorbidities, specifically GERD, must be weighed with complication risks when selecting conversion procedures, especially in high-risk patients.

\section{References}

1. Aarts EO, Dogan K, Koehestanie P, et al. (2014) Long-term results after laparoscopic adjustable gastric banding: A Mean fourteen year follow-up study. Surg Obes Relat Dis 10: 633-640.

2. Kindel T, Martin E, Hungness E, et al. (2014) High failure rate of the laparoscopic-adjustable gastric band as a primary bariatric procedure. Surg Obes Relat Dis 10:1070-1075.

3. Lewis CS, Varma AK, Hamdorf JM (2016) Comparison of safety between 1-stage and 2-stage surgery: From laparoscopic adjustable gastric banding to laparoscopic sleeve gastrectomy. Surg Obes Relat Dis 12: 976-983.

4. Gangemi A, Russel S, Patel K, et al. (2018) Conversion to laparoscopic sleeve gastrectomy after failure of laparoscopic gastric band: A systematic review of the literature and cost considerations. Obes Res Clin Pract 12: 416-420.

5. Janik MR, Rogula TG, Mustafa RR, et al. (2019) Safety of revision sleeve gastrectomy compared to roux-Y gastric bypass after failed gastric banding analysis of the MBSAQIP. Ann Surg 269: 299-303.

6. Brethauer SA, Kothari S, Sudan R, et al. (2014) Systematic review on reoperative bariatric surgery: American society for metabolic and bariatric surgery revision task force. Surg Obes Relat Dis 10: 952-972.

7. https://asmbs.org/resources/estimate-of-bariatric-surgery-numbers.

8. Mann JP, Jakes AD, Hayden JD, et al. (2015) Systematic review of definitions of failure in revisional bariatric surgery. Obes Surg 25: 571-574.

9. Mechanick JI, Youdim A, Jones DB, et al. (2013) Clinical practice guidelines for the perioperative nutritional, metabolic, and nonsurgical support of the bariatric surgery patien-2013 Update: Cosponsored by american association of clinical endocrinologists, the obesity society, and american society for metabolic \&amp; bariatric surgery. Endocr Pract 19: 337-372.

10. Schneck AS, Lazzati A, Audureau E, et al. (2016) One or two steps for laparoscopic conversion of failed adjustable gastric banding to sleeve gastrectomy: A Nationwide French study on 3357 morbidly obese patients. Surg Obes Relat Dis 12: 840-848.

11. Carandina S, Genser L, Bossi M, et al. (2017) Laparoscopic sleeve gastrectomy after failed gastric banding: Is It really effective? Six years of follow-up. Surg Obes Relat Dis 13: 1165-1173.

12. Dietch ZC, Schirmer BD, Hallowell PT (2017) Simultaneous conversion of gastric band to sleeve gastrectomy is associated with increased postoperative complications: An analysis of the American College of Surgeons National Surgical Quality Improvement Program. Surg Endosc 31: 5228-5233.

13. Pencovich N, Lahat G, Goldray O, et al. (2017) Safety and outcome of laparoscopic sleeve gastrectomy following removal of adjustable gastric banding: Lessons from 109 patients in a single center and review of the literature. Obes Surg 27: 1266-1270.

14. Hii MW, Lake AC, Kenfield C, et al. (2012) Laparoscopic conversion of failed gastric banding to roux-en-Y gastric bypass. Shortterm follow-up and technical considerations. Obes Surg 22: 1022-1028.

15. Shoar S, Saber AA (2017) Long-term and midterm outcomes of laparoscopic sleeve gastrectomy versus Roux-en-Y gastric bypass: A Systematic review and meta-analysis of comparative studies. Surg Obes Relat Dis 13: 170-180.

16. Creange C, Jenkins M, Pergamo M, et al. (2018) Gastric Band Conversion to Roux-en- $Y$ Gastric Bypass Shows Greater Weight Loss than Conversion to Sleeve Gastrectomy: 5-year Outcomes. Surg Obes Relat Dis 14: 1531-1536.

17. paniolas K, Bates AT, Docimo S, et al. (2017) Single stage conversion from adjustable gastric banding to sleeve gastrectomy or Roux-en-Y gastric bypass: An Analysis of 4875 patients. Surg Obes Relat Dis 13: 1880-1884.

18. Noria SF, Grantcharov T (2013) Biological effects of bariatric surgery on obesity-related comorbidities. Can J Surg 56: 47-57.

19. Cardoso L, Rodrigues D, Gomes L, et al. (2017) Short- and longterm mortality after bariatric surgery: A systematic review and meta-analysis. Diabetes Obes Metab 9: 1223-1232.

20. Lager CJ, Esfandiari NH, Luo Y, et al. Metabolic Parameters, Weight Loss, and Comorbidities 4 Years After Roux-en-Y Gastric Bypass and Sleeve Gastrectomy. Obes Surg 28: 3415-3423.

21. El-Hadi M, Birch DW, Gill RS, et al. (2014) The effect of bariatric surgery on gastroesophageal reflux disease. Can J Surg 57: 13944.

22. Khan A, Kim A, Sanossian C, et al. (2016) Impact of obesity treatment on gastroesophageal reflux disease. World J Gastroenterol 22: 1627-1638.

23. Viscido G, Gorodner V, Signorini F, et al. (2018) Laparoscopic sleeve gastrectomy: Endoscopic findings and gastroesophageal reflux symptoms at 18 -month follow-up. J Laparoendosc Adv Surg Tech A 28: 71-77.

24. Dakour Aridi H, Asali M, Fouani T, et al. (2017) Gastroesophageal reflux disease after laparoscopic sleeve gastrectomy with concomitant hiatal hernia repair: An unresolved question. Obes Surg 27: 2898-2904.

DOI: $10.36959 / 367 / 445$

Copyright: (C) 2020 Howell RS, et al. This is an open-access article distributed under the terms of the Creative Commons Attribution License, which permits unrestricted use, distribution, and reproduction in any medium, provided the original author and source are credited. 